\title{
Knowledge Management in the Semiconductor Industry: Dispatches from the Front Line
}

\author{
Brian Donnellan \\ Brian Donnellan, Centre for Innovation and Structural Change, \\ National University of Ireland, Galway \\ brian.donnellan@nuigalway.ie
}

\begin{abstract}
The semiconductor industry is characterized by products with a high level of intellectual property content, long product development cycles, designed by very scarce engineering talent. Because of the knowledge-intensive nature of the development process barriers to market entry are extremely strong. The foundation of many semiconductor company's successes has been the research and development of new products, with a range of products being sold to thousands of customers in many different horizontal and vertical markets. There are a number of knowledge management challenges facing corporations in the semiconductor industry: how to improve the sharing of knowledge and best practices across the organization, how to quickly develop solutions to technical problems and hence reduce time-to-market, and how to accelerate innovation rates by bringing diverse views and experience to bear. This paper will describe some of these challenges. The research is based on a case study of a leading semiconductor company with worldwide operations.
\end{abstract}

Keywords: Knowledge Management, Knowledge Management Systems, New Product Development, Innovation.

\section{Introduction}

Leading proponents of Knowledge Management (KM) in the semiconductor industry include Hewlett Packard (HP) and Texas Instruments (TI). Thomas Davenport describes how Hewlett Packard have adopted a strategic approach to KM [1]. He describes KM initiatives in using Lotus Notes. HP established three different 'knowledge bases' for educators in the company. Three projects involving HP's Product Processes Organisation were described. One involved competitor information for HP's Components group. The goal of the second project was to create a web-based interface to primary and secondary research information. The third system managed international marketing information.

Carla ODell describes how Texas Instruments (TI) attempted to identify and transfer internal best practices [2]. In 1993 TI's former CEO, president and chairman of the board, the late Jerry Jenkins initiated a project to create a common methodology and common language which would be used to provide best practices across the company. One of the earliest and simplest facilitators of knowledge sharing was the standardisation of the company on Microsoft applications in the early 1990's, which enabled individuals to share documents through attachments and file transfer. In 1995, 200 Texas Instruments employees worldwide were set up to use a 'Best Prac- 
tice Knowledge Base' in Lotus Notes. A practice is documented in the system with a title, a short narrative and contact information. Each practice is categorised for retrieval by: (i) quality criteria (ii) process and (iii) keywords.

This paper will examine current KM issues in the semiconductor industry. The analysis will be grounded in the experiences of a leading company in the industry. The organizational context for this study is a semiconductor new product development group comprised of 430 staff members. The group is based in Ireland and is part of a larger multi-national corporation. The corporation is a world leader in the design, manufacture, and marketing of high-performance analog, mixed-signal and digital signal processing integrated circuits (ICs) used in signal processing applications. Founded in 1965, it employs approximately 8,800 people worldwide.

Table 1. Structure of this Paper

\begin{tabular}{l|l|}
\hline Section & Topic \\
\hline 1 & $\begin{array}{l}\text { Introduction: This section will give some background and motivation for } \\
\text { this research. }\end{array}$ \\
\hline 2 & $\begin{array}{l}\text { The Semiconductor Industry: This section will give an overview of the } \\
\text { semiconductor industry and indicate some of the KM challenges faced by } \\
\text { firms in that industry. }\end{array}$ \\
\hline 3 & $\begin{array}{l}\text { KM Challenges faced by firms in the Semiconductor Industry: There are } \\
\text { some KMS challenges faced in the semiconductor industry. They will be } \\
\text { described in this section. }\end{array}$ \\
\hline 4 & $\begin{array}{l}\text { Examples of KMS in the Semiconductor Industry: Companies have re- } \\
\text { sponded to the KMS challenges associated with NPD by developing portfo- } \\
\text { lios of KMS applications. Some examples will be given. }\end{array}$ \\
\hline 5 & Summary, Conclusions \\
\hline
\end{tabular}

\section{The Semiconductor Industry}

The semiconductor industry has grown considerably over the last 30 years to the point where it is now constitutes over $\$ 100 \mathrm{~B}$ in worldwide sales. This growth has been achieved in a very dynamic, turbulent, operating environment. Specifically, the following dynamics are currently impacting semiconductor firms:

- A difficult and uncertain economic environment, where many customers continue to experience flat or declining growth

- Increased outsourcing of manufacturing by semiconductor manufacturers and their customers

- Increased complexity, fragmentation and globalization of markets

- Ever compressing product lifecycles in many product segments

- Continued rapid technology evolution and the ability of manufacturers to respond to and invest in these changes

Consequently, semiconductor firms are faced with many business challenges:

- The selling process for component suppliers is becoming increasingly complex in this multi-tier, multi-party and global ecosystem.

- Manufacturers are under pressure to win more designs than ever. 
- Lack of visibility into the demand chain makes opportunity management, account management and forecasting inherently difficult.

- Managing the complex interactions with their distribution partners, including managing sales reporting, inventory, and liabilities.

- Pressure to decrease costs without sacrificing customer or partner loyalty

To address these challenges, semiconductor companies need new strategies to assure their success or even survival during these times of increased competition and economic uncertainty.

\section{KM Challenges Faced by Firms in the Semiconductor Industry}

The "resource-based" view of the firm emphasizes the importance of a firm's resources, including intellectual capital, as its source of sustainable competitive advantage. Grant states "what distinguishes the Knowledge Economy from previous economies is the sheer accumulation of knowledge by society, the rapid pace of innovation and, most important, the advent of digital technologies that have had farreaching implications for the sources of value in the modern economy" [4]. He identifies four aspects of management practice in NPD organizations that are being impacted by the emergence of the Knowledge Economy:

\section{a) Property rights in knowledge}

Recognition of the value of proprietary knowledge has increased the amount of intellectual property legislation by legislatures and judicial systems over the past two decades. The enforcement of intellectual property in the form of patents, copyrights, and trademarks has become a central asset-management activity [5].

\section{b) Accelerating knowledge creation and application}

Companies engaged in NPD have struggled to shorten their NPD cycles. For example, the fundamental force behind Intel's sustained success is its "time pacing" - the time pacing of NPD though continual minor innovation with periodic "mid-life kickers", together with a nine-month fabrication cycle [6].

\section{c) Converting tacit into explicit knowledge}

Kogut and Zander coined the term "paradox of replication" to describe where the codification of knowledge required for internal replication may also facilitate replication of that knowledge by other firms [7]. The challenge facing KM practitioners appears to be how to build barriers to external replication through linking internal systems to knowledge that cannot be replicated by outsiders [8].

\section{d) Competing for standards}

Over the last two decades, there has been a change in attitude towards the role of industry standards. Firms are now more willing to sacrifice short-term financial gains for long-term benefits derived from standardization processes. These strategies can imply that firms have to form collaborative projects with customers, competitors and government agencies to achieve a standardization goal. These types of projects, by their nature, place a lot of emphasis on KM capabilities.

Ramesh and Tiwana analysed the NPD process for a Personal Digital Assistant operating system, and went on to develop a prototype system to support collaborative 
NPD [9]. Court, Culley et al. investigated the use of information in NPD teams and reported on the use of information technology to support the NPD process [10]. They analyzed the methods by which the NPD team members retrieve, apply and subsequently transfer their information. A significant finding was that even though team members have access to IS tools and services, they still preferred to use manual and verbal methods of communication and information retrieval. These preferred formats may suggest that computer information accessing and storage is still at the infancy stage and therefore used with some reluctance by design teams. A key challenge appeared to the researchers to be the extensive use of personal information stores and the absence of easy-to-use indexing systems.

Anderson et al. looked at the design activity in Rank Xerox and illustrated how collaborative, inter-actional, and organizational ordering are not addressed by the information technology infrastructure in the Design Dept. at Rank Xerox [11]. Adler et al. argued for a process-oriented approach to NPD and used a case study of a fictitious company, which represented a composite of a number of companies studied by Adler [12]. He claimed that the process oriented approach, which had crossfunctional teams as a central element, led to the creation of best practice templates which in turn led to greater efficiencies in NPD. Van de Ven and Polley empirically demonstrate how the early stages of NPD projects can be accounted for by using principles drawn from chaos theory - providing potential future insight into the front end of NPD efforts that traditionally have proven elusive [13]. Scott proposed a framework that decomposed the NPD process into three phases and then classified the types of knowledge and IS appropriate for each phase [14].

\subsection{Demands for Increased Productivity in New Product Development}

NPD processes may have short product and process life cycles. These cycles are getting shorter and they are compressing the available time window for recouping the expenses associated with NPD. This places a premium on the ability to effectively capture knowledge created during the process so that it can be re-used in the next generation of products to reduce development time. This capture-reuse cycle is a key enabler for productivity improvements in the design phase of NPD. Underlying the growth has been a fundamental driver of market growth called Moore's Law. Moore's Law is an historical observation by Intel executive, Gordon Moore, that the market demand (and semiconductor industry response) for functionality per chip (bits, transistors) doubles every 1.5 to 2 years. He also observed that MPU performance $($ clock frequency $(\mathrm{MHz}) \times$ instructions per clock $=$ millions of instructions per second (MIPS)) also doubles every 1.5 to 2 years. Moore's Law has been a consistent macro trend and key indicator of successful leading-edge semiconductor products and companies for the past 30 years.

However it has been estimated that productivity $($ where productivity $=$ dollar value-add per unit of engineering effort in the U.S. Semiconductor Industry $1986-$ 1995, source: U.S. census and bureau of labour and statistics) among electronic design engineers doubles every 36 months [15]. The competitive pressure to improve productivity and thereby reduce the NPD cycle time is huge. Since the challenges associated with capturing and reusing knowledge are, by their nature, knowledge management challenges - this is one of the key KM challenges being posed by NPD. 
KMS responses to this challenge range from the application of knowledge "codification" systems to knowledge "personalization" systems [16].

\subsection{Internal Knowledge Transfer}

Today's NPD organizations need to facilitate knowledge transfer across internal organizational boundaries. The drive to enable this knowledge transfer may stem from any one of a number of factors: the existence of "virtual teams" that are geographically dispersed, the re-organization of NPD activities from a linear to a concurrent model or the need for stronger communication flow between organizational units that had been disconnected heretofore e.g. sales and manufacturing.

\subsubsection{Virtual NPD Teams}

NPD organizations can be distributed across geographical boundaries. The NPD activity that spans these centers requires the teams to share their knowledge across team boundaries. It also creates a need for KMS infrastructure to support and promote knowledge sharing. The challenges posed by distributed teams may arise from cultural differences. The appreciation of cultural differences across geographically dispersed teams may be a key factor in the success of those teams. There are at least four ways in which culture influences the behaviours central to knowledge sharing in NPD teams:

a) Culture shapes assumptions about what knowledge is and which knowledge is worth managing. Sackman empirically demonstrated four different kinds of cultural knowledge: "dictionary" knowledge, "directory" knowledge, "recipe" knowledge and "axiomatic" knowledge [17]. Hedlund and Nonaka contrasts U.S. and Japanese practices of managing knowledge [18]. The basis for the contrast is the cultural difference between U.S. and Japanese firms.

b) Culture defines the relationships between individual and organizational knowledge, determining who is expected to control specific knowledge, as well as who must share it and who can hoard it. This relationship is influenced by what some researchers refer to as the presence of an atmosphere of "care" in a company. "Care" can be characterized by an active empathy, access to help and lenience in judgement. Von Krogh and Roos stress that knowledge nurturing and creating organizations should be caring organizations [19]. Culture can also promote unique attitudes toward communication and information, which in extreme cases can restrict knowledge transfer to the point of organizational demise as demonstrated by Brown and Starkey [20].

c) Culture creates the context for social interaction that determines how knowledge will be shared in particular situations. Reducing harsh bureaucratic structures and increasing informal communication may empower creativity and innovation by promoting spontaneity, experimentation and freedom of expression [21]. This culture entails an almost total removal of many of the values that underpinned the reengineering and "right sizing" management culture of the early 1990's. For example, knowledge cultures value a "fat" middle management layer for professional support and a tolerance for the functional inefficiency that a messy, chaotic creative process implies [22]. 
d) Culture shapes the processes by which the new knowledge with its accompanying uncertainties is created, legitimated, and distributed in organizations. In this context Hayduk developed a framework of organizational practices to foster knowledge sharing that is based on sensitivities to the national culture in which a firm finds itself located [23].

\subsubsection{Cross-Functional Collaboration}

Many NPD projects require cross-functional collaboration. The nature and importance of this collaboration is described by Wheelwright and Clark as follows:

"Outstanding product development requires effective action from all of the major functions in the business. From engineering one needs good designs, well-executed tests, and high quality-proto-types; from marketing, thoughtful product positioning, solid customer analysis, and well-thought-out product plans; from manufacturing, capable processes, precise cost estimates and skilful pilot production and ramp-up. Great products and processes are achieved when all of these activities fit well together. The firm must develop the capability to achieve integration across the functions in a timely and effective way." p.165 [24]

The patterns of communication are described in Table 2. The ends of the spectra represent opposites in integration. On the left is a communication pattern that is sparse, infrequent, one-way, and late. One the right, the communication is rich, frequent, reciprocal, and early. This is the preferred mode of communication for NPD organizations because collaborating engineers meet face to face with their colleagues early in the design process and share preliminary ideas with sketches, models, and notes.

Table 2. Communication between Functional Groups in NPD [24]

\begin{tabular}{lll}
\hline Communication Dimension & Range of Choice & \\
\hline Richness of Media & $\begin{array}{l}\text { Sparse: documents, } \\
\text { computer networks }\end{array}$ & $\begin{array}{l}\text { Rich: face-to-face, } \\
\text { models }\end{array}$ \\
\hline Frequency & Low: One-shot, batch & $\begin{array}{l}\text { High: piece-by-piece, } \\
\text { on-line, intensive }\end{array}$ \\
\hline Directions & One-way: monologue & Two-way: dialogue \\
\hline Timing & $\begin{array}{l}\text { Late: completed work, ends } \\
\text { the process }\end{array}$ & $\begin{array}{l}\text { Early: preliminary, } \\
\text { begins the process }\end{array}$ \\
\hline
\end{tabular}

\subsection{Cross-Institutional Collaboration}

Cross-institutional collaboration is also becoming quite common in NPD processes. The need for this type of collaboration arises when organizations seek to collaborate with sources of knowledge, which are external to it. For instance a firm may want to work with an internationally recognized centre-of-excellence in an academic institution with which it has no formal relationship. Cases where NPD teams want to work closely with external standards organizations are also becoming more prevalent. In such cases knowledge has to be combined from participants across multiple collaborating organizations. 


\subsection{Transient Team Membership}

NPD teams are staffed with people who may possess much sought-after skills and expertise. Consequently there can be high turnover rates in NPD organizations, as firms compete for staff with highly rated R\&D experience. The resulting transient existence of teams results in a reduction in organizational knowledge unless there is a repository for knowledge rather than a dependence on knowledge that is solely situated in the minds of individuals.

There is also a requirement that some staff turnover should exist for NPD teams to be effective. The rate of movement of staff members across organizational boundaries has been shown to have an effect on NPD team output. Katz explored the relationship between the mean tenure of NPD teams, the degree of external communication, and performance [25]. In his study of 50 NPD teams in a large American corporation, he found that initially group performance increased with increasing mean tenure of the group, but this relationship reversed and performance dropped off after five years. The decline in performance was significantly correlated with a decline in external communication and a growth in so-called Not-Invented-Here (NIH) behavior [26].

\section{Examples of KM Initiatives in the Semiconductor Industry}

Semiconductor firms have responded to the KMS challenges associated with NPD by adopting a dual approach. On one hand, a portfolio of KMS applications are being developed. On the other hand, peer reviews that are an integral part of the stage-gate process are being leveraged as knowledge-sharing opportunities.

\subsection{NPD Meta-knowledge}

"Conventional explanations view learning as a process by which a learner internalizes the knowledge, whether "discovered," "transmitted" from others, or "experienced in interaction" with others." (p.47) [27]. However, before one can initiate such a process, whether through discovery or interaction, there must be a mechanism by which people can easily find out what knowledge is being created in the organization and by whom. The knowledge being sought is, in fact, knowledge about knowledge or "meta-knowledge" [28], [29].

Meta-knowledge attempts to provide answers to questions such as "Where can I get information about a particular technical topic? How can I find out more about this topic? Is there work in progress in this organization on this topic?" KMS applications address these challenges by making it easy for members of the technical staff to publish and locate technical reviews, notes, articles etc. - items which previously may have required several emails and phone calls to track down.

\subsection{Catalogs}

A "Catalog", in this context, is an application that generates a list of previously designed products in the product development community. The catalog would enable product development staff to quickly find out if products were previously designed that were similar to those currently under development. The entries are created and owned by the product development staff. Each entry in the catalog represents is a 
potentially reusable circuit design. Catalog entries, depending on their utility, are potential candidates for inclusion in a repository. The problems that were identified in the NPD process that were to be addressed by catalogs are:

(a) a lack of awareness of what previously designed circuit blocks had been created and might be available for reuse in future projects

(b) a mechanism by which product development staff could easily make their products more easily "discovered" by members of the product development organization outside of their own organization unit

\subsection{NPD Design Repositories}

A "Repository", in this context, provides a store of previously design products that could be reused throughout the corporation. Each of the repository's elements has an extensive support kit associated with it i.e. thorough documentation, contextual information about previous usage, data formats compatible with existing NPD systems, validation data, interface information, etc. The goal of the repository is to provide a library of robust and supported reusable circuit designs available for download, obtained from both internal and external sources. The repository contains previously designed products packaged in a format suitable to delivery as intellectual property to either internal groups or external groups (or both). The repository is a structured repository for formal knowledge containing previously used circuits that were internally developed and externally procured circuits that may also be re-used in future products. Its purpose corresponds, generally, to what Hansen termed a "codification" strategy where the value of the repository lies in "connecting people with reusable codified knowledge" [16] or to what Swan termed a "cognitive" strategy where the primary function of the repository is to codify and capture knowledge so that the knowledge can be recycled (Swan, Newell et al. 1999).

\subsection{Implications for Practitioners}

The underlying philosophical approach to the firm's knowledge management initiative was that knowledge management should be integrated into the daily work of people in the organization. A number of steps were taken in the development and implementation of the knowledge management process that were designed to promote the integration of the process into the daily working of the organization:

- Focus: progress in the initiative was systematically monitored in a balanced scorecard that captured different facets of organizational performance

- Alignment: the placing of the knowledge management process in a framework with other key business processes promoted alignment with those processes. Decisions were made on all the business processes in a collective manner with some mutual adjustment where necessary to achieve an overall consistency in strategic direction.

- Instruments: There were specific KM services and enabling technologies provided to promote and facilitate knowledge management. These included the types of KMS applications described elsewhere in this paper.

- Communities: Communities-Of-Practice were set up which proved to be powerful mechanisms for disseminating knowledge across organizational boundaries. 


\section{Summary and Conclusions}

This paper has provided an overview of the knowledge management challenges faced by firms in the semiconductor industry. The industry was characterized by products with a high level of intellectual property content, long product development cycles, designed by very scarce engineering talent. Examples were provided of knowledge management applications designed to overcome some of these challenges. The research was based on a case study of a leading semiconductor company with worldwide operations.

\section{References}

1. Davenport, T.H., Ten Principles of Knowlede Management and Four Case Studies. Knowledge and Process Management, 1997. 4(3): p. 187-208.

2. O'Dell, C. and G. Jackson, If Only We Knew What We Know: Identification and Transfer of Internal Best Practices. California Management Review, 1998. 40(3): p. 154-174.

3. Moore, G. Keynote Address. in International Solid State Circuits Conference. 2003. San Francisco.

4. Grant, R., Shifts in the World Economy: The Drivers of Knowledge Management, in Knowledge Horizons: The Present and the Promise of Knowledge Management, C. Despres and D. Chauvel, Editors. 2000, Butterworth-Heinemann: Boston. p. 27-55.

5. Grindley, P. and D. Teece, Managing intellectual capital: licensing and cross-licensing in semiconductors and electronics". California Management Review, 1997. 39 (Winter): p. $8-41$.

6. Brown, S.L. and K.M. Eisenhardt, Competing on the Edge: Strategy as Structured Chaos. 1998, Boston: Harvard Business Press.

7. Kogut, B. and U. Zander, Knowledge of the Firm, Combinative Capabilities, and the Replication of Technology. Organisation Science, 1992. 3(3): p. 383-397.

8. Schultze, U. Investigating The Contradictions In Knowledge Management. in IFIP. 1998.

9. Ramesh, B. and A. Tiwana, Supporting Collaborative Process Knowledge Management in New Product Development Teams. Decision Support Systems, 1999. 27(1-2): p. 213-235.

10. Court, A.W., S.J. Culley, and C.A. McMahon, The Influence of Information Technology in New Product Development: Observations of an Empirical Study of the Access of Engineering Design Information. International Journal of Information Management, 1997. 17(5): p. 359-375.

11. Anderson, B., G. Button, and W. Sharrock. Supporting The Design Process Within An Organisational Context. in ECSCW'93. 1993. Milan.

12. Adler, P.S., et al., Getting the Most Out of Your Product Development Process, in Harvard Business Review. 1996. p. 134-152.

13. van de Ven, A.H. and D. Polley, Learning While Innovating. Organisation Science, 1992. 3(1): p. 92-116.

14. Scott, J.E. The Role of Information Technology in Organizational Knowledge Creation for New Product Development. in Second Americas Conference on Information Systems. 1996.

15. Collett, R., A Strategic Analysis of the Emerging Market for IP and The Role Of Design Re-Use 1998-2002, in Presentation to VSIA Membership. 1998.

16. Hansen, M., N. Nohria, and T. Tieney, What's your strategy for managing knowledge? Harvard Business Review, 1999. March-April(1999): p. 106-116.

17. Sackmann, S.A., Cultures and Subcultures: An Analysis of Organisational Knowledge. Administrative Science Quarterly, 1992. 37(1): p. 140-161. 
18. Hedlund, G. and I. Nonaka, Models of Knowledge Management in the West and Japan, in Implementing Strategic Process: Change, Learning and Cooperation, P.L.e. al., Editor. 1993, Basil Blackwell: Oxford. p. 117-144.

19. von Krogh, G. and J. Roos, eds. Managing Knowledge : Perspectives on Cooperation and Competition. 1996, Sage: London.

20. Brown, A.D. and K. Starkey, The Effect of Organisational Culture on Communication and Information. Journal of Management Studies, 1994. 31(6): p. 808-828.

21. Graham, A.B. and V.G. Pizzo, A question of balance: case studies in strategic knowledge management. European Management Journal, 1996. 14(4): p. 338-346.

22. Baskerville, R. and J. Pries-Heje. Managing knowledge capability and maturity. in IFIP 8.2 and 8.6 Joint Working Conference on Information Systems. 1998. Helsinki.

23. Hayduk, H. Organizational Culture Barriers to Knowledge Management. in Association for Information Systems - Americas Conference. 1998.

24. Wheelwright, S. and K. Clark, Revolutionalizing Product Development. 1992, New York: Simon and Schuster Inc.

25. Katz, R., The effects of group longevity on project communication and performance. Administrative Science Quarterly, 1982. 27: p. 81-104.

26. Brown, S.L. and K.M. Eisenhardt, Product Development: Past Research, Present Findings, and Future Directions. Academy of Management Review, 1995. 20(2): p. 343-378.

27. Lave, J. and E. Wenger, Situated learning: Legitimate peripheral participation. 1991, Cambridge: Cambridge University Press.

28. Swanstrom, E., MetaKnowledge and MetaKnowledgebases, in The Knowledge Management Handbook, J. Liebowitz, Editor. 1999, CRC Press: London.

29. Kehal, M., Searching For An Effective Knowledge Management Framework. Journal Of Knowledge Management Practice, 2002(February, 2000). 